\title{
A smartphone based surface plasmon resonance imaging (SPRi) platform for on-site biodetection
}

\author{
Hasan Guner $^{\mathrm{a}}$, Erol Ozgur ${ }^{\mathrm{a}}$, Guzin Kokturk ${ }^{\mathrm{b}}$, Mehmet Celik ${ }^{\mathrm{c}}$, Elif Esen ${ }^{\mathrm{b}}$, Ahmet E. Topal $^{\mathrm{a}}$, \\ Sencer Ayas ${ }^{\mathrm{d}}$, Yildiz Uludag ${ }^{\mathrm{b}}$, Caglar Elbuken ${ }^{\mathrm{a}}$, Aykutlu Dana ${ }^{\mathrm{a}, *}$ \\ a UNAM, Institute of Materials Science and Nanotechnology, Bilkent University, Ankara, 06800, Turkey \\ b UEKAE-BILGEM, The Scientific and Technological Research Council of Turkey (TUBITAK), Gebze, Kocaeli, 41470, Turkey \\ ' Department of Computer Engineering, Middle East Technical University, Ankara, 06800, Turkey \\ d Canary Center at Stanford for Cancer Early Detection, Department of Radiology, Stanford School of Medicine, Palo Alto, CA 94304, USA
}

\section{A R T I C L E I N F O}

\section{Article history:}

Received 2 July 2016

Received in revised form 5 August 2016

Accepted 10 August 2016

Available online 10 August 2016

\section{Keywords:}

Plasmonics

Surface plasmon resonance imaging

Optical discs

Mobile sensing

Biosensors

Optical sensor

\begin{abstract}
A B S T R A C T
We demonstrate a surface plasmon resonance imaging platform integrated with a smartphone to be used in the field with high-throughput biodetection. Inexpensive and disposable SPR substrates are produced by metal coating of commercial Blu-ray discs. A compact imaging apparatus is fabricated using a 3D printer which allows taking SPR measurements from more than 20.000 individual pixels. Real-time bulk refractive index change measurements yield noise equivalent refractive index changes as low as $4.12 \times 10^{-5}$ RIU which is comparable with the detection performance of commercial instruments. As a demonstration of a biological assay, we have shown capture of mouse IgG antibodies by immobilized layer of rabbit anti-mouse (RAM) IgG antibody with nanomolar level limit of detection. Our approach in miniaturization of SPR biosensing in a cost-effective manner could enable realization of portable SPR measurement systems and kits for point-of-care applications.
\end{abstract}

(c) 2016 Published by Elsevier B.V.

\section{Introduction}

The wide use of mobile phones all across the world created significant opportunities for healthcare applications using mobile devices. Improvement of healthcare services requires democratization of the services with higher quality and lower cost. Early diagnosis, close monitoring, patient comfort are some of the concerns that healthcare providers are striving to improve on. The development of lab-on-a-chip platforms in the last two decades brought several examples of novel platforms that can be used for rapid diagnosis of widespread diseases. Portability, short turn-around-time, cost-efficiency and connectivity are some of the critical assets that successful devices should possess. The advancements in such areas enabled the use of lab-on-a-chip systems as on-site or point-of-care systems not only for remote or resource-limited settings, but also for home-monitoring of elderly population at developed countries.

One of the main bottlenecks in transforming the lab-on-a-chip systems into point-of-care diagnostic devices is the requirement to miniaturize and combine several off-chip components. The mar-

\footnotetext{
* Corresponding author.

E-mail address: aykutlu@unam.bilkent.edu.tr (A. Dana).
}

riage of the lab-on-a-chip systems with mobile phones was the tipping point that yielded a plethora of integrated screening and diagnostic devices. Mobile phones provide powerful CPUs, touch screen displays, advanced connectivity features as well as high pixel-count, sensitive cameras and integrated light sources. Therefore, the use of mobile phones for applications requiring optical detection is an interesting and rapidly developing field of research. For instance, immunodiagnostic assays, lateral flow assays, microscopic imaging, flow cytometry, colorimetric detection, photonic crystal and surface plasmon resonance (SPR) based biosensing have been demonstrated using mobile phone platforms [1-12]. In this study, to the best of our knowledge we present the first surface plasmon resonance imaging on a smartphone.

SPR biosensing is a popular method for quantitative analysis and characterization of biomolecular interactions [13-15]. SPR provides label-free and real-time detection of binding events with high sensitivity. Surface plasmons are electromagnetic waves propagating along and evanescently decaying away from the metal/dielectric interface. This field confinement around the boundary makes plasmon resonance coupling condition extremely sensitive to the local refractive index changes caused by specific adsorption of target analytes onto the molecular probes residing on the metal surface. Recently, some studies have demonstrated SPR sensing using smartphone. Preechaburana et al. reported angle- 
resolved SPR chemical detection using an SPR coupler attached on the smartphone screen utilizing the illumination from the screen [8]. In order to develop a more practical system, Liu et al. [9] and Bremer et al. [10] showed the use of optical fibers for SPR detection on cell phones. Both of these systems used the back-side LED and camera of the cell phone as the light source and the sensor, respectively. Roche et al. demonstrated localized SPR sensing on cell phone with increased sensitivity using gold nanoparticles and nanorods [11]. Dutta et al. reported localized SPR (LSPR) sensing on cell phone for biomolecular detection and measuring size variation of metal nanoparticles [12]. All of these studies, demonstrate single-spot or 1D spatially resolved SPR sensing, which limits their applicability for high-throughput and multiplexed detection. In this article, we demonstrate SPR imaging or 2D SPR sensing on a cell phone, unveiling the potential of multi-analyte detection as well as implementation of array-based advanced biochemical analysis using a low-cost, integrated platform (See Supplementary material Table S1 for detailed comparison with previous studies).

SPR imaging is superior to single-spot SPR since it can be used for detection of multiple analytes in a single sample for panel assays. This leads to significant benefits in terms of cost and measurement time. Similarly, the multiple sensing points on the sensor can be used for detection of the analyte at several sample dilutions which is critical for serial dilution assays. Also, image-based bioanalytical detection helps the operator to view the results at once and interpret them more easily. Additionally, image-based 2D sensing can provide replicated measurements on the same sensor chip together with controls that leads to higher reliability, precision and on-chip self-calibration. On-chip control and self-calibration are especially critical for point-of-care sensing applications that suffer from high error rates due to varying operating conditions and the wide range of user levels. We believe the demonstration of such advanced biochemical techniques using mobile phones and cost-effective sensors will lead to a paradigm shift in the global healthcare market. Implementation of advanced detection applications on continuously improving feature-rich mobile phones will pave the way towards highly sensitive diagnosis tools reaching to the people from all socio-economic levels.

Here, we present surface plasmon resonance imaging on a smartphone. We have developed very low-cost grating coupled SPR sensor chips using off-the-shelf optical storage discs. Additionally, we designed a compact optical system, using a 3D-printed apparatus that hosts the LED source, collimator, bandpass filter, linear polarizer, beamsplitter plate and an external imaging lens which can be easily attached to the smartphone. We employed a silver/gold $(\mathrm{Ag} / \mathrm{Au})$ bilayer structure coated on the periodic corrugations of Blu-ray discs in order to perform plasmon resonance imaging at the central region of visible spectrum $\left(\lambda_{\mathrm{r}} \sim 500 \mathrm{~nm}\right)$ under normal incidence illumination in aqueous environment. This allowed the optimal use of the CMOS sensor of the smartphone while maintaining high sensitivity, chemical stability and biological affinity [16-20]. A microfluidic channel is placed on the bimetallic layer for controlled plumbing of the liquids. The use of Blu-ray discs and standard metal deposition techniques together with the low-cost microfluidic channel resulted in significant cost-reduction which can allow the system to be used for applications requiring disposable SPR sensors.

\section{Materials and methods}

\subsection{Smartphone attachment for surface plasmon resonance imaging}

An optical attachment was developed which converts smartphone into a real-time surface plasmon resonance imaging platform based on intensity interrogation mechanism. A Samsung I8552 Galaxy Win was used as the smartphone. The prototype accessory was fabricated out of polylactic acid (PLA) filament using a 3D printer (MakerBot Replicator 2). Optical configuration of the imaging platform is schematically illustrated in Fig. 1a. Light emitting from a $520 \mathrm{~nm}$ LED source is coupled to a multimode fiber optic cable (acting as a spatial filter) and collimated by a fiber optic collimator. A bandpass interference filter $\left(\lambda_{C}=520 \mathrm{~nm}\right.$, $\Delta \lambda_{\text {FWHM }}=10 \mathrm{~nm}$ ) is used to narrow the spectral bandwidth of illumination. Collimated beam of light becomes transverse magnetic (TM) polarized passing through a linear polarizing filter sheet and is directed onto the sensor surface at normal incidence by reflecting from a beamsplitter plate. Light reflecting off the sensor surface passes through the beamsplitter plate and is focused on the smartphone's camera sensor by an external plastic imaging lens (focal length $=8 \mathrm{~mm}$ ). Imaging spot covers approximately 160 pixels in diameter which corresponds to more than 20.000 individual pixels, although the camera is capable of video recording at $720 \times 480$ pixels resolution. Imaging resolution is calculated as $12 \mu \mathrm{m} /$ pixel. Only green channel of RGB image is analyzed since it has the highest spectral responsivity at the operation wavelength. The attachment measures $143 \times 75 \times 44 \mathrm{~mm}^{3}$ and weighs $215 \mathrm{~g}$ including $2 \mathrm{AAA}$ batteries (Fig. 1b).

An Android application software was developed to analyze image data, report intensity changes and estimate analyte concentration in the field use (Fig. 1c). When the app is initiated, zoomed in view of the imaging spot is displayed continuously at the background. First, region of interests (ROI) on the sensor surface are defined by tapping menu button and entering ROI parameters in the settings menu. To estimate analyte concentrations from intensity changes, predetermined calibration line parameters are set in slope, intercept and unit fields. Time averaging feature can be activated by entering the number of consecutive frames to be averaged. "Reference", "Dark" and "Baseline" buttons are used to take and store reference, dark and baseline intensity values, respectively, as described in Subsection 2.3. Averaged intensity changes and estimated analyte concentrations for each ROI segment are displayed separately on the right side of the screen when the "Start recording" box is checked. Unchecking the "Start recording" item stops recording and saves measurement data in a text file. Screen captures from application software are presented in Supplementary material Fig. S1.

\subsection{Design and fabrication of SPRi sensor chips}

Optical storage discs can be exploited as grating coupled surface plasmon resonance sensors by metal coating, thanks to the periodic corrugations [21,22]. Depending on the disc type and refractive index of the surrounding medium, surface plasmons can be excited at wavelengths ranging from ultraviolet to near infrared spectral regions. SPR substrates produced from Blu-ray discs (BD) (grating period $\Lambda=320 \mathrm{~nm}$, grating depth $d=20 \mathrm{~nm}$ ) exhibit plasmon resonances within the visible spectrum when illuminated at normal incidence in aqueous medium, as indicated by the reflectance spectra calculations (Fig. S2). Numerical calculations are performed using a commercial software (PCGrate) which employs modified integral method (MIM) [23]. PCGrate allows calculation of diffraction efficiencies of multilayered 1-D gratings. Calculation mode should be switched from normal to resonance mode for SPR coupler simulations. Polarization of the incident beam is set as non-polarized in order to examine the reflectance spectra for both transverse electric (TE) and transverse magnetic (TM) polarized illuminations. Surface profile of the grating on BD is defined as sine-trapezoidal using built-in geometrical tools. Complex refractive indices of gold and silver are extrapolated from previously taken experimental data [24]. According to the simu- 
(a)
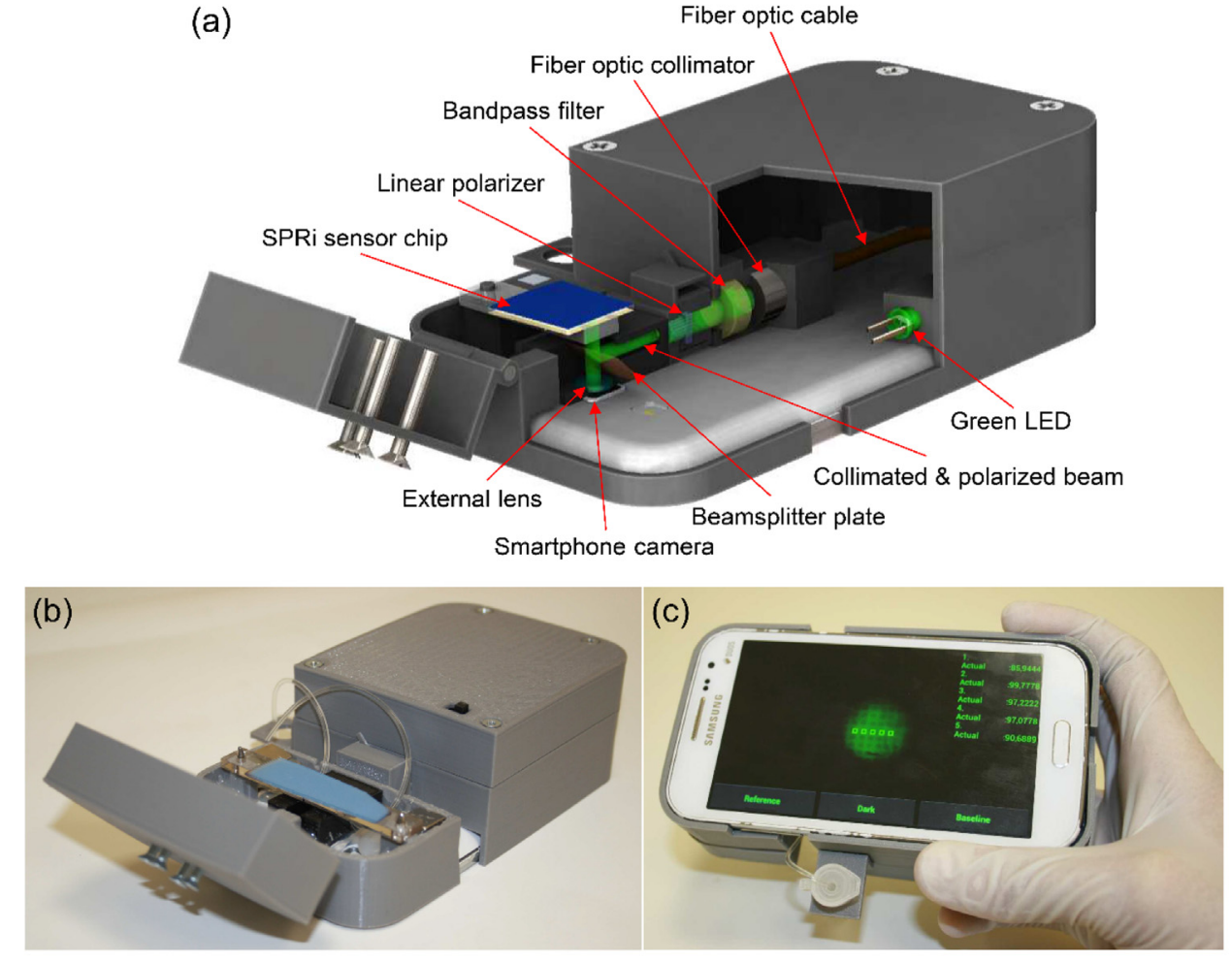

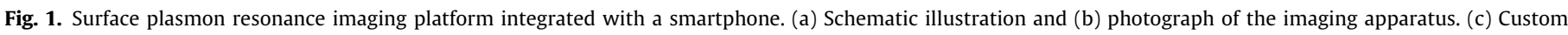
developed smartphone application for real-time and on-site monitoring of multiple sensing spots.

lation results, silver coated $\mathrm{BD}$ exhibits sharp plasmon resonance around $500 \mathrm{~nm}$ wavelength, whereas gold coated BD does not display surface plasmon resonant behavior at normal incidence of excitation due to its intrinsic absorption characteristics at this portion of the spectrum. However, gold has superior properties over silver in terms of chemical stability and adequacy for further surface functionalization chemistry for biosensing applications. In order to overcome this dichotomy, we came up with a hybrid solution in which thick $(>80 \mathrm{~nm})$ silver coating at the bottom acts as a plasmon excitation layer and thin $(<10 \mathrm{~nm})$ gold film on top functions as a surface for the subsequent surface modification, as well as extending the shelf life of the sensor chip. The thicknesses of Ag and Au layers are optimized using numerical simulations. Silver layer thickness above $80 \mathrm{~nm}$ is found to yield almost same SPR reflectance spectra with semi-infinite silver layer configuration as shown in Fig. S3. As the thickness of the top gold layer is increased, on the other hand, plasmon resonance curve degrades and loses its sharpness as shown in Fig. S4. Thus, gold coating thickness is kept as $2 \mathrm{~nm}$ in order to satisfy both sensitivity and surface chemistry requirements. Apart from the previous studies which use the $\mathrm{Ag} / \mathrm{Au}$ bimetallic structure to increase sensitivity [16-20], we used this configuration to obtain a response below $520 \mathrm{~nm}$ which is not possible with the commonly used single gold layered couplers.

Blu-ray discs (BD) are converted to plasmonic surfaces by peeling off the transparent thin protective coating on top of the disc with tweezer after cutting a notch on the side of the disc, followed by metallization using thermal vacuum evaporation (Vaksis Thermal Evaporator). First, a thin germanium layer is deposited to act as an adhesion layer as well as to reduce the surface roughness of subsequent silver coating $[25,26]$. Germanium $(3 \mathrm{~nm})$, silver $(80 \mathrm{~nm})$ and gold $(2 \mathrm{~nm})$ metal layers are deposited at $0.4 \AA / \mathrm{s}, 0.6-1.0 \AA / \mathrm{s}$ and $0.2 \AA / s$ growth rates, respectively, under 0.005 mTorr chamber pressure. Schematic illustration and SEM image of a Ag/Au bilayer coated BD structure are shown in Fig. 2.
Sensitivity performance of a bimetallic BD based SPR sensor is investigated using numerical simulations. The spectral bulk refractive index sensitivity, $S_{\lambda \mathrm{BC}}$ is calculated as $316 \mathrm{~nm} / \mathrm{RIU}$. The ratio of the change in resonance wavelength to the change in the thickness of layer formed by the adsorption of molecules onto the sensor surface gives the spectral surface coating sensitivity, $S_{\lambda s c}$, and it is calculated as $0.7 \mathrm{~nm} / \mathrm{nm}$ for a coating material with refractive index, $\mathrm{n}=1.45$.

Blu-ray discs as described here allow easy replication of our work by others without needing any infrastructure investment. Moreover, sensor surfaces can be produced at lower costs on a large scale following the manufacturing processes of Blu-ray discs. In fact roll-to-roll printing of gratings is demonstrated [27], which can be used as an alternative to Blu-ray discs. It is important to note that planar sensor surfaces that are fabricated using any of the processes mentioned above are completely compatible with our optical readout configuration.

A low-cost microfluidic channel is fabricated using an extremely simple, yet very effective method based on laser cutting of a $3 \mathrm{~mm}$ thick transparent acrylic plate and a double sided adhesive tape (3M 468MP Adhesive Transfer Tape) (Fig. S5). Both channel geometry and holes for inlet and outlet are defined by a high power $\mathrm{CO}_{2}$ laser cutting system (Epilog Zing 24). Channel geometry is defined on the double sided tape at $8 \mathrm{~W}$ laser power and $100 \%$ scanning speed. Channel frame, inlet and outlet holes are defined on the acrylic plate at $30 \mathrm{~W}$ laser power and $15 \%$ scanning speed. First, double sided tape is bond onto the acrylic plate. Then, acrylic plate and $\mathrm{BD}$ chip are bond together tightly by applying mechanical pressure for half a minute. Schematic illustration of a flow cell integrated SPRi sensor chip is shown in Fig. 2. Tygon tubings (Cole-Palmer) are connected to the inlet and outlet of the flow cell. Epoxy adhesive (Bison Epoxy 5 Minutes) is used to completely seal the tubings at the inlet and outlet connections. The volume of a flow cell is 8 

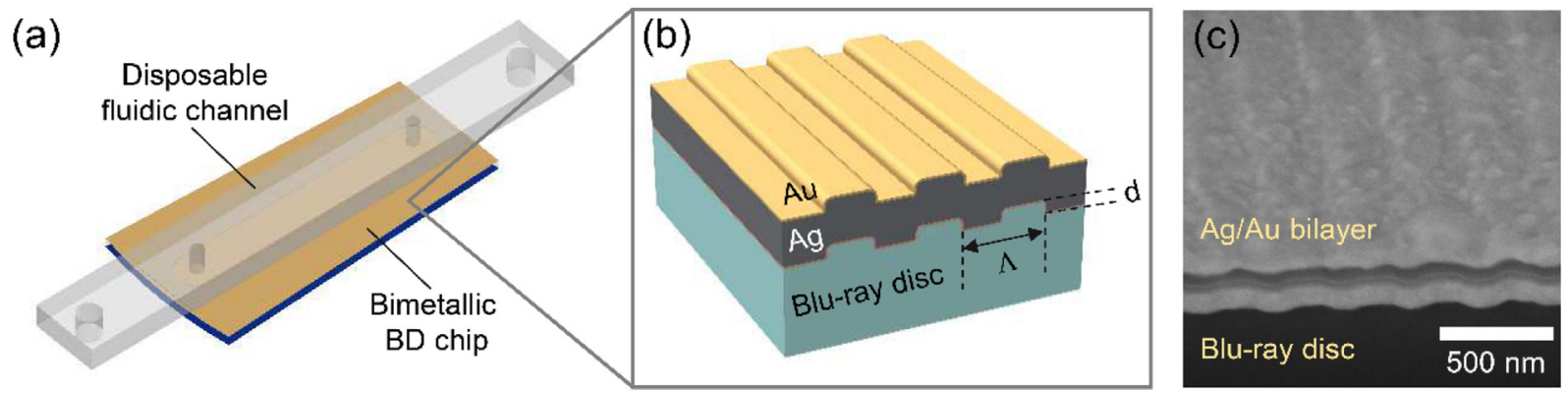

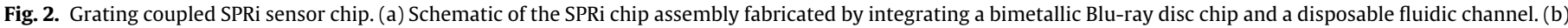

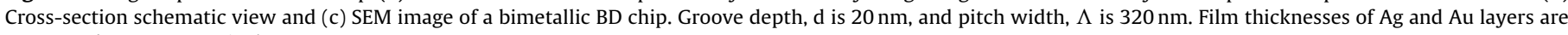
$80 \mathrm{~nm}$ and $2 \mathrm{~nm}$, respectively.

microliters $\left(16 \times 5 \times 0.1 \mathrm{~mm}^{3}\right)$. An external peristaltic pump is used for controlled plumbing of the liquids through the channel.

\subsection{Measurement protocol}

In a typical measurement, an SPRi sensor chip is plugged into the sample holder unit. Next, LED light source is turned on and polarizing filter is adjusted to transverse electric (TE) polarization where electric field of the transmitted light is parallel to the grating lines of the sensor surface. Inlet port of the flow cell is connected to the pump and outlet port is connected to a waste tube. Flow cell is filled with the running buffer solution. Orientation of the sample with respect to the incident light beam is finely tuned by adjusting set screws behind the sample holder cover until the illumination spot displayed on the touchscreen has a circular shape and gets located at the center of the screen. Having the sample properly aligned, surface image is recorded under TE polarized illumination to be taken as normalization reference. Then the light source is turned off and screen image is captured as dark reading. For the rest of the measurement, the light source is turned on and polarizing filter is adjusted to TM polarization. Before performing the assay protocol, surface image is recorded to establish baseline reading while the sample is in its bare state and surrounded by buffer solution. Intensity change at each pixel is calculated by taking the difference between actual and baseline intensities. Time and area averaging options are provided in the application software to obtain enhanced signal during the course of the measurement. From surface activation to the capture of analyte molecules, the whole assay protocol can be monitored in real-time.

\subsection{Experimental setup for wavelength interrogation of plasmon resonance}

Reflectance spectra of surface plasmon resonances excited on the sensor chips are probed in real-time using a normal incidence SPR spectroscopy setup (Fig. S6). White light emitted from a high power broadband LED source driven by a stable DC current source (Keithley 2400 SourceMeter) is coupled to a multimode fiber optic cable and collimated by a collimation lens at the output of the fiber. Collimated beam of light passes through an adjustable linear polarizer, an adjustable iris to narrow down the beam diameter, and a non-polarizing beam splitter cube. Polarized and narrowed beam is reflected off the sensor surface, directed by the beam splitter towards the spectrometer fiber optic components, collected by a fiber coupling lens and analyzed by a spectrometer (Ocean Optics Maya 2000 Pro). Each reflectance spectrum measurement is taken at $20 \mathrm{~ms}$ of integration time. The spectral position of the resonance dip is probed using centroid algorithm [28]. Resonance wavelength shift noise of a spectrogram averaged over 75 spectra is calculated as $1.5 \mathrm{pm}$. Taking the experimental spectral bulk refractive index sensitivity, $\mathrm{S}_{\lambda \mathrm{BE}}$ as $356 \mathrm{~nm} / \mathrm{RIU}$ for BD SPR sensors, the minimum detectable bulk refractive index change, $\sigma_{\mathrm{nBE}}$ is found as $4.2 \times 10^{-6}$ RIU for the wavelength interrogation setup.

\subsection{Optimization of the illumination wavelength}

The illumination wavelength $\left(\lambda_{i}\right)$ of the imaging platform which yields the highest intensity sensitivity was identified by measuring reflectance spectra for varying refractive indices of the surrounding liquid medium (Fig. S7). Liquid solutions with refractive indices ranging from 1.335 to 1.365 were prepared by dissolving glycerol $(\mathrm{n}=1.474)$ in deionized water $(\mathrm{n}=1.335)$ at varying concentrations. The highest bulk refractive index sensitivity was found to be about $800 \% /$ RIU at the wavelength of $520 \mathrm{~nm}$ for TDK BD-R substrate. Both resonance wavelength shift and intensity change $\left(\lambda_{i}=520 \mathrm{~nm}\right)$ show linear dependence on the refractive index change within the $1.335<\mathrm{n}_{\mathrm{d}}<1.365$ range.

\section{Results and discussion}

\subsection{Microarray imaging of $\mathrm{Ag} / \mathrm{Au}$ bimetallic sensing spots}

We have performed SPR imaging of $\mathrm{Ag} / \mathrm{Au}$ bimetallic microspot array under bulk dielectric media with changing refractive indices. The bimetallic microarray structure was fabricated using conventional optical lithography processes in addition to metal deposition steps. The diameter of each spot is $110 \mu \mathrm{m}$ and the spacing between adjacent spots is $130 \mu \mathrm{m}$. First, we have recorded video of illuminated sensor surface at 30 frames/s during the successive flow of glycerol solutions with increasing refractive indices. Each 200 consecutive video frames were averaged in order to enhance the signal-to-noise ratio. As the refractive index of the surrounding medium gets higher, plasmon resonance curve shifts towards longer wavelengths resulting in darker imaging spots under TM polarized illumination. Fig. 3a shows green channel view of an averaged RGB image of a microspot sensor array in 20\% v/v glycerol solution environment $\left(n_{d}=1.3596\right)$. SPRi difference images were generated by taking TE images as normalization reference, and TM images in pure water medium as background (Fig. 3b). Line profile of a row of microarray reveals the uniformity of the reflectivity change across the spots (Fig. 3c). Average reflectivity change of spots were measured at each glycerol concentration level from $1 \%$ to $25 \%$ v/v (See Fig. S8 for time resolved responses of individual sensing spots during fluid exposures). The glycerol calibration curve for $4 \times 4$ spots located within the rectangular frame shown in Fig. 3a exhibits high linearity $\left(R^{2}=0.9951\right)$ within a dynamic range of $22200 \times 10^{-6}$ RIU between $n_{d}=1.3354$ and 1.3576 (Fig. $3 d$ ). The error bars shown in Fig. 3d show twice the standard deviation (2SD) of the measured value demonstrating the low intra-sensor variability between 16 spots. The slope of the calibration curve which gives 
(a)

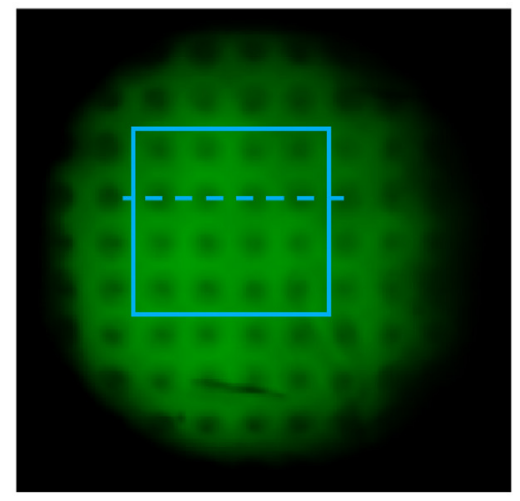

(c)

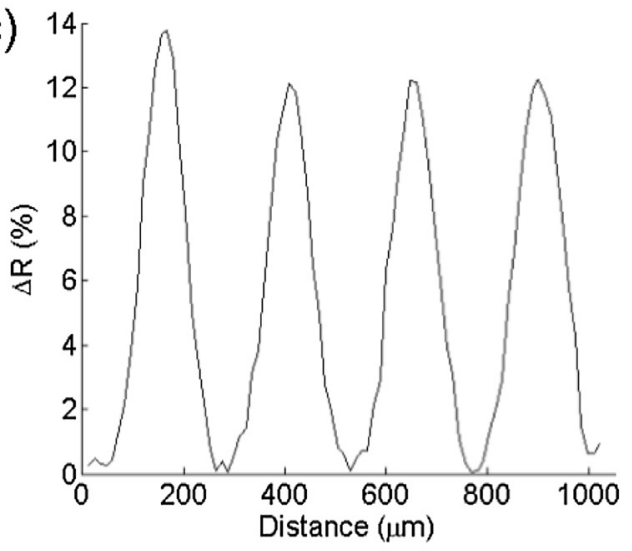

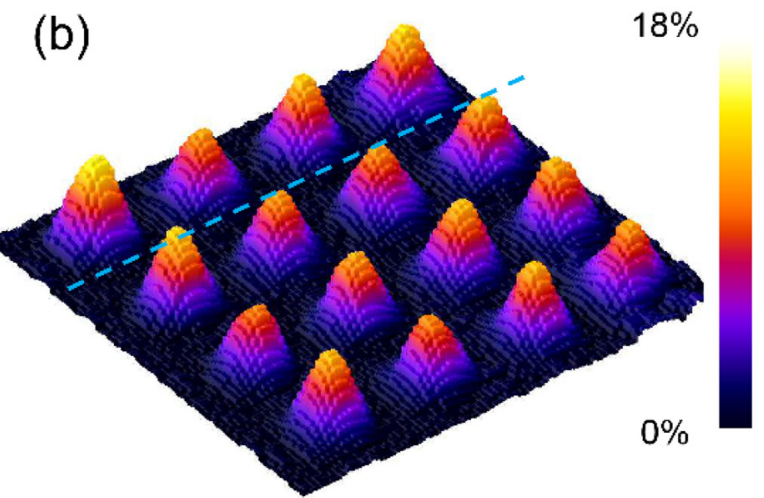

(d)

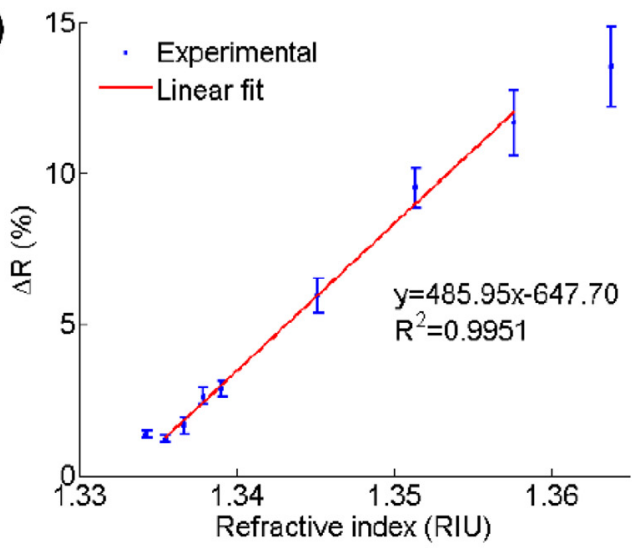

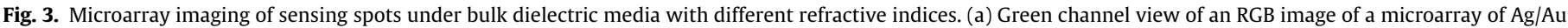

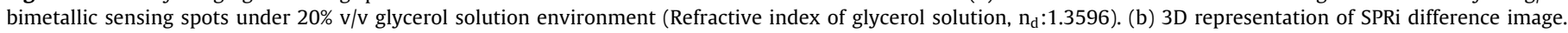

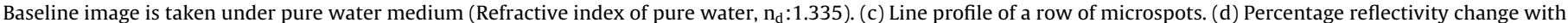

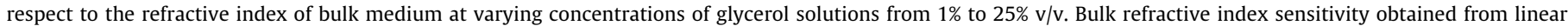

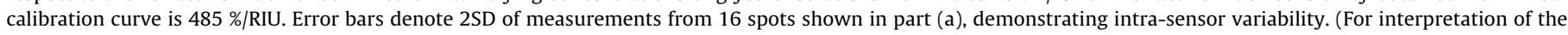
references to colour in this figure legend, the reader is referred to the web version of this article.)

the experimental reflectivity bulk refractive index sensitivity, $S_{\mathrm{RBE}}$ was found as $485 \% /$ RIU. Intensity noise of a single pixel in terms of percentage reflectivity, $\sigma_{\mathrm{R}}$ was found as $0.2 \%$, whereas averaging a rectangular area of 10 -by-10 pixels $\left(120 \times 120 \mu \mathrm{m}^{2}\right)$ reduces the reflectivity noise down to $0.02 \%$ level. Thus, the minimum detectable bulk refractive index change, $\sigma_{\mathrm{n}}$ over a 10 -by-10 pixels area is calculated as $4.12 \times 10^{-5}$ RIU for the smartphone platform. The low cost Ag/Au bimetallic BD SPR structure exhibits relatively low refractive index sensitivity as compared to the previously reported SPR grating, prism and waveguide couplers [13,29-32]. However, since the noise level is also reduced by area averaging, overall resolution of the system is comparable with the previously demonstrated studies.

\subsection{Real-time monitoring of bovine serum albumin (BSA) adsorption}

We have taken time-resolved intensity change measurements in response to the protein adsorption on the sensor surface. Bovine serum albumin (BSA) is known to adsorb onto the gold surface forming a 4-7 nm thick monolayer [33]. $1 \mathrm{mg} / \mathrm{mL}(15 \mu \mathrm{M}) \mathrm{BSA}$ (Sigma Aldrich) dissolved in $10 \mathrm{mM}$ phosphate buffer solution (PBS, $\mathrm{pH}=7.4$ ) was injected through the flow cell for $5 \mathrm{~min}$ after a thorough PBS wash. Following the formation of self-assembled monolayer on the gold coating, the sensor surface was cleaned by PBS wash again. SPRi difference image of arbitrarily defined 4-by-4 rectangular region of interests after the BSA adsorption is shown in Fig. 4a. Average reflectivity change of 16 ROIs over time is as shown in Fig. $4 \mathrm{~b}$ and the steady-state reflectivity difference is found as $4.37 \pm 0.53 \%$. Resonance wavelength shift caused by the BSA adsorption was measured using SPR wavelength interrogation setup repeating the same BSA protocol. The resonance dip shifts from $503.8 \mathrm{~nm}$ to $507 \mathrm{~nm}$ as shown in Fig. 4c. Reflectivity at $520 \mathrm{~nm}$ decreases by $6.44 \%$ from $52.46 \%$ to $46.02 \%$, in agreement with imaging results.

We have performed kinetic analysis of the reaction based on a simple interaction model explained in the Supplementary material. The association rate, $\mathrm{k}_{\mathrm{a}}$ of the BSA-Au complex formation is calculated as $1150 \mathrm{M}^{-1} \mathrm{~s}^{-1}$, with the assumption that the dissociation rate, $\mathrm{k}_{\mathrm{d}}$ is negligibly small as can be deduced from the sensorgram curve after second PBS injection.

\subsection{Demonstration of a biodetection assay}

As an exemplary biodetection experiment, capture of mouse IgG antibody by immobilized layer of rabbit anti-mouse (RAM) IgG antibody protocol was implemented as direct assay. First, surface of a thin gold film coated BD chip was cleaned by argon plasma treatment. Sensor surface was coated with self-assembled monolayer (SAM) of 11-mercaptoundecanoicacid (11-MUDA) by immersing the chip in $2 \mathrm{mM}$ ethanol solution of mercaptoundecanoic acid overnight followed by rinsing with ethanol and water, and drying in the fume hood. Then, BD chip was integrated with a flow cell. Ethanol (70\%) and PBS solutions were injected 
(a)

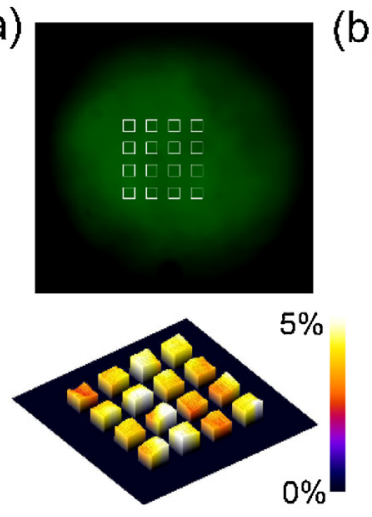

(b)

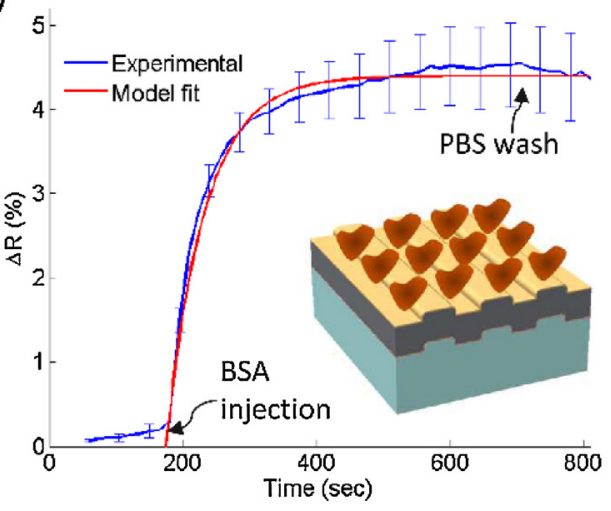

(c) 80

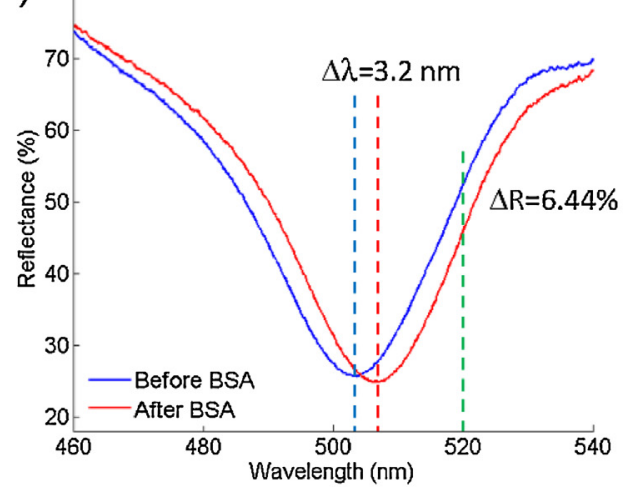

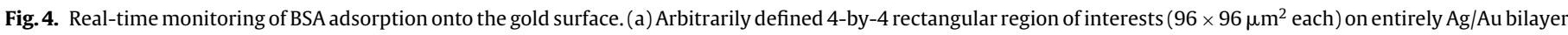

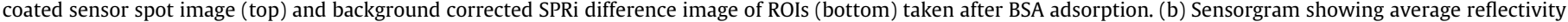

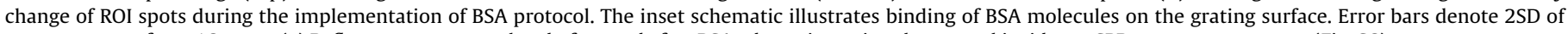
measurements from 16 spots. (c) Reflectance spectra taken before and after BSA adsorption using the normal incidence SPR spectroscopy setup (Fig. S6).
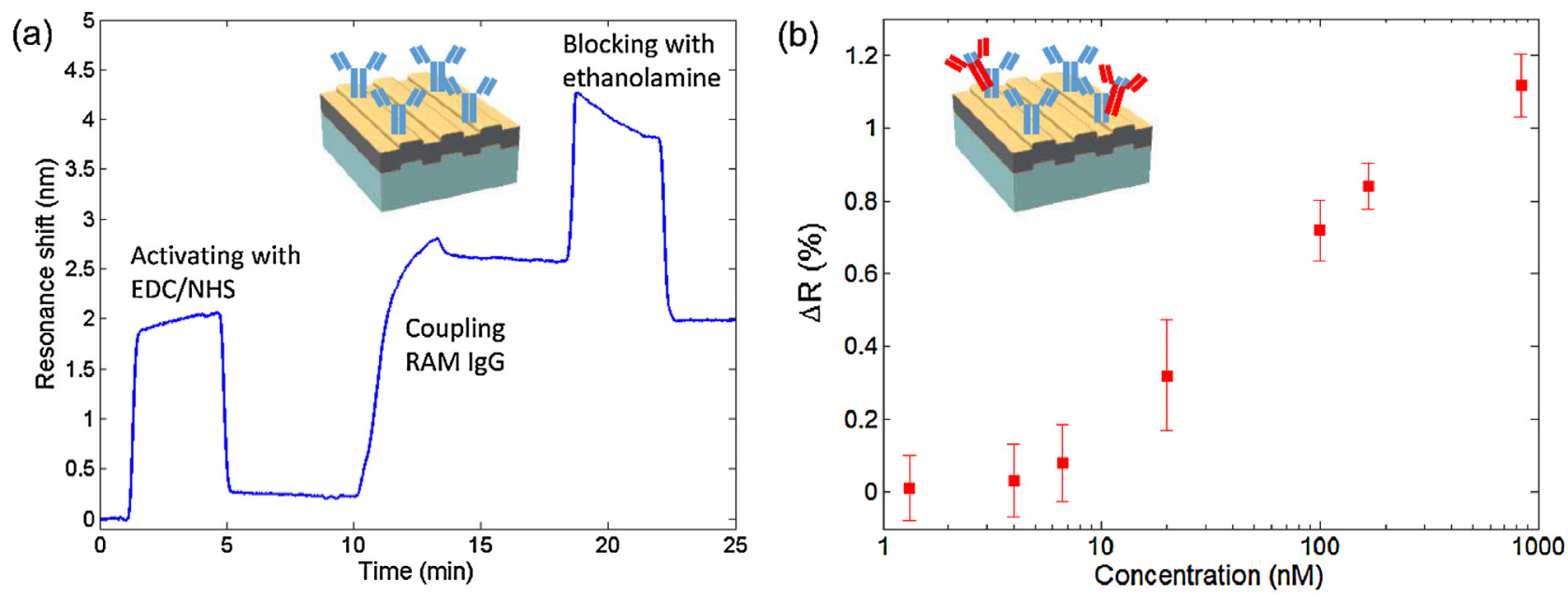

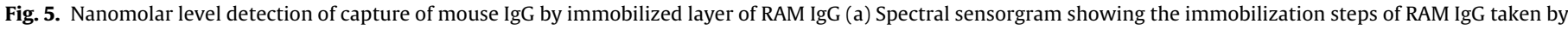

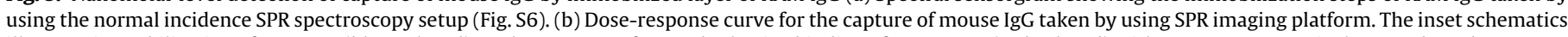

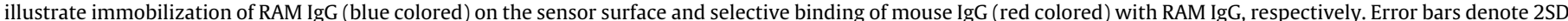
of measurements from 3 spots. (For interpretation of the references to colour in this figure legend, the reader is referred to the web version of this article.)

onto the surface at $100 \mu \mathrm{L} / \mathrm{min}$ flow rate for further cleaning. Surface of the 11-MUDA SAM layer was activated by the exposure of $400 \mathrm{mM}$ 1-ethyl-3-(3 dimethylaminopropyl)-carbodiimide (EDC) and $100 \mathrm{mM}$-hydroxysuccinimide (NHS) mixture (1:1) at $100 \mu \mathrm{L} / \mathrm{min}$ flow rate for $4 \mathrm{~min}$. Both reagents were prepared in distilled water and mixture was prepared just before use. Diluted RAM IgG $(50 \mu \mathrm{g} / \mathrm{mL})$ in $0.1 \mathrm{mM}$ sodium acetate buffer solution was injected onto the activated sensor surface at $50 \mu \mathrm{L} / \mathrm{min}$ flow rate for 3 min. RAM IgG antibodies were immobilized on the activated surface by amine coupling chemistry. Following the RAM Fc immobilization, non-reacted NHS esters were capped by the exposure of $1 \mathrm{M}$ ethanolamine solution at $100 \mu \mathrm{L} / \mathrm{min}$ flow rate for $4 \mathrm{~min}$ to prevent non-specific analyte bindings. Sensor surface was cleaned by PBS wash for 5 min after each step. The whole immobilization process from surface activation to blocking step was probed in realtime using the wavelength interrogation setup (Fig. 5a).

SPR chip immobilized with RAM IgG is taken out the wavelength interrogation setup and plugged into the smartphone attachment. Mouse IgG solutions at concentrations ranging from $1.33 \mathrm{nM}$ to $830 \mathrm{nM}$ were injected successively at $50 \mu \mathrm{L} / \mathrm{min}$ flow rate for $5 \mathrm{~min}$. Intensity changes of individual pixels at three distinct locations on the sensor surface is shown in Fig. 5b. Dose-response curve reveals that nanomolar level detection of antibody analyte is achievable within a dynamic range from a few nanomolars to micromolar concentration.

\section{Conclusion}

In this work, we demonstrated the use of a smartphone as a hand-held surface plasmon resonance imaging biosensor with high sensitivity. Developing an attachable imaging accessory from inexpensive optical components and 3D printed parts, and using easy-to-implement procedures for fabricating miniaturized sensor chips integrated with flow cells from extremely cheap substrates like optical storage discs, we offer a promising detection platform that enables biosensing in the field with high level of portability and affordable cost. The capability to perform parallel assays in a short amount of time allows the use of the instrument for point-of-care applications where monitoring of multiple parameters is desirable.

\section{Acknowledgement}

This work was supported by the State Planning Agency of the Turkish Republic Project UNAM. 


\section{Appendix A. Supplementary data}

Supplementary data associated with this article can be found, in the online version, at http://dx.doi.org/10.1016/j.snb.2016.08.061.

\section{References}

[1] S.K. Vashist, O. Mudanyali, E.M. Schneider, R. Zengerle, A. Ozcan, Cellphone-based devices for bioanalytical sciences, Anal. Bioanal. Chem. 406 (2014) 3263-3277, http://dx.doi.org/10.1007/s00216-013-7473-1.

[2] Y. Lu, W. Shi, J. Qin, B. Lin, Low cost, portable detection of gold nanoparticle-labeled microfluidic immunoassay with camera cell phone, Electrophoresis 30 (2009) 579-582, http://dx.doi.org/10.1002/elps. 200800586

[3] O. Mudanyali, S. Dimitrov, U. Sikora, S. Padmanabhan, I. Navruz, A. Ozcan, Integrated rapid-diagnostic-test reader platform on a cellphone, Lab Chip 12 (2012) 2678-2686, http://dx.doi.org/10.1039/c2lc40235a.

[4] D.N. Breslauer, R.N. Maamari, N.A. Switz, W.A. Lam, D.A. Fletcher, Mobile phone based clinical microscopy for global health applications, PLoS One 4 (2009) e6320, http://dx.doi.org/10.1371/journal.pone.0006320.

[5] H. Zhu, S. Mavandadi, A.F. Coskun, O. Yaglidere, A. Ozcan, Optofluidic fluorescent imaging cytometry on a cell phone, Anal. Chem. 83 (2011) 6641-6647, http://dx.doi.org/10.1021/ac201587a.

[6] L. Shen, J. a. Hagen, I. Papautsky, Point-of-care colorimetric detection with a smartphone, Lab Chip 12 (2012) 4240, http://dx.doi.org/10.1039/c2lc40741h.

[7] D. Gallegos, K.D. Long, H. Yu, P.P. Clark, Y. Lin, S. George, et al., Label-free biodetection using a smartphone, Lab Chip 13 (2013) 2124-2132, http://dx. doi.org/10.1039/c3lc40991k.

[8] P. Preechaburana, M.C. Gonzalez, A. Suska, D. Filippini, Surface plasmon resonance chemical sensing on cell phones, Angew. Chem. Int. Ed. Engl. 51 (2012) 11585-11588, http://dx.doi.org/10.1002/anie.201206804.

[9] Y. Liu, Q. Liu, S. Chen, F. Cheng, H. Wang, W. Peng, Surface plasmon resonance biosensor based on smart phone platforms, Sci. Rep. 5 (2015) 12864, http:/ dx.doi.org/10.1038/srep12864.

[10] K. Bremer, B. Roth, Fibre optic surface plasmon resonance sensor system designed for smartphones, Opt. Express 23 (2015) 17179-17184, http://dx. doi.org/10.1364/OE.23.017179.

[11] P.J.R. Roche, S. Filion-Côté, M.C.K. Cheung, V.P. Chodavarapu, A.G. Kirk, A camera phone localised surface plasmon biosensing platform towards low-cost label-free diagnostic testing, J. Sens. 2011 (2011) 1-7, http://dx.doi. org/10.1155/2011/406425.

[12] S. Dutta, K. Saikia, P. Nath, Smartphone based LSPR sensing platform for bio-conjugation detection and quantification, RSC Adv. 6 (2016) 21871-21880, http://dx.doi.org/10.1039/C6RA01113F.

[13] B. Liedberg, C. Nylander, I. Lunström, Surface plasmon resonance for gas detection and biosensing, Sens. Actuators 4 (1983) 299-304, http://dx.doi. org/10.1016/0250-6874(83)85036-7.

[14] J. Homola, Present and future of surface plasmon resonance biosensors, Anal. Bioanal. Chem. 377 (2003) 528-539, http://dx.doi.org/10.1007/s00216-0032101-0.

[15] J. Homola, Surface plasmon resonance sensors for detection of chemical and biological species, Chem. Rev. 108 (2008) 462-493, http://dx.doi.org/10.1021/ cr068107d.

[16] B.H. Ong, X. Yuan, S.C. Tjin, J. Zhang, H.M. Ng, Optimised film thickness for maximum evanescent field enhancement of a bimetallic film surface plasmon resonance biosensor, Sens. Actuators B Chem. 114 (2006) 1028-1034, http:// dx.doi.org/10.1016/j.snb.2005.07.064.

[17] L. Xia, S. Yin, H. Gao, O. Deng, C. Du, Sensitivity enhancement for surface plasmon resonance imaging biosensor by utilizing gold-silver bimetallic film configuration, Plasmonics 6 (2011) 245-250, http://dx.doi.org/10.1007/ s11468-010-9195-y.

[18] Y. Chen, R.S. Zheng, D.G. Zhang, Y.H. Lu, P. Wang, H. Ming, et al., Bimetallic chips for a surface plasmon resonance instrument, Appl. Opt. 50 (2011) 387-391, http://dx.doi.org/10.1364/AO.50.000387.

[19] C.-T. Li, K.-C. Lo, H.-Y. Chang, H.-T. Wu, J.H. Ho, T.-J. Yen, Ag/Au bi-metallic film based color surface plasmon resonance biosensor with enhanced sensitivity, color contrast and great linearity, Biosens, Bioelectron 36 (2012) 192-198, http://dx.doi.org/10.1016/j.bios.2012.04.016.

[20] T.T. Ehler, L.J. Noe, Surface plasmon studies of thin silver/gold bimetallic films, Langmuir 11 (1995) 4177-4179, http://dx.doi.org/10.1021/la00010a088.

[21] B. Kaplan, H. Guner, O. Senlik, K. Gurel, M. Bayindir, A. Dana, Tuning optical discs for plasmonic applications, Plasmonics 4 (2009) 237-243, http://dx.doi. org/10.1007/s11468-009-9099-X.

[22] B. Turker, H. Guner, S. Ayas, O.O. Ekiz, H. Acar, M.O. Guler, et al., Grating coupler integrated photodiodes for plasmon resonance based sensing, Lab Chip 11 (2011) 282-287, http://dx.doi.org/10.1039/c0lc00081g.

[23] I. International Intellectual Group, PCGrate-S, 2011.
[24] E.D. Palik, Handbook of Optical Constants of Solids, 1985.

[25] Logeeswaran Vj, N.P. Kobayashi, M.S. Islam, W. Wu, P. Chaturvedi, N.X. Fang, et al., Ultrasmooth silver thin films deposited with a germanium nucleation layer, Nano Lett. 9 (2009) 178-182, http://dx.doi.org/10.1021/nl8027476.

[26] W. Chen, M.D. Thoreson, S. Ishii, A.V. Kildishev, V.M. Shalaev, Ultra-thin ultra-smooth and low-loss silver films on a germanium wetting layer, Opt. Express 18 (2010) 5124-5134, http://dx.doi.org/10.1364/OE.18.005124.

[27] S.H. Ahn, L.J. Guo, High-Speed roll-to-roll nanoimprint lithography on flexible plastic substrates, Adv. Mater. 20 (2008) 2044-2049, http://dx.doi.org/10. 1002/adma.200702650.

[28] G.G. Nenninger, M. Piliarik, J. Homola, Data analysis for optical sensors based on spectroscopy of surface plasmons, Meas. Sci. Technol. 13 (2002) 2038-2046, http://dx.doi.org/10.1088/0957-0233/13/12/332.

[29] D.C. Cullen, R.G.W. Brown, C.R. Lowe, Detection of immuno-complex formation via surface plasmon resonance on gold-coated diffraction gratings, Biosensors 3 (1987) 211-225, http://dx.doi.org/10.1016/0265928X(87)85002-2.

[30] C. Nylander, B. Liedberg, T. Lind, Gas detection by means of surface plasmon resonance, Sens. Actuators 3 (1982) 79-88, http://dx.doi.org/10.1016/02506874(82)80008-5.

[31] Z. Wang, Z. Cheng, V. Singh, Z. Zheng, Y. Wang, S. Li, et al., Stable and sensitive silver surface plasmon resonance imaging sensor using trilayered metallic structures, Anal. Chem. 86 (2014) 1430-1436, http://dx.doi.org/10.1021/ ac402126k.

[32] L. Song, Z. Wang, D. Zhou, A. Nand, S. Li, B. Guo, et al., Waveguide coupled surface plasmon resonance imaging measurement and high-throughput analysis of bio-interaction, Sens. Actuators B Chem. 181 (2013) 652-660, http://dx.doi.org/10.1016/j.snb.2013.01.096.

[33] D.C. Carter, J.X. Ho, Structure of serum albumins, Adv. Protein Chem. 45 (1994) 153-203, http://dx.doi.org/10.1016/S0065-3233(08)60640-3.

\section{Biographies}

Hasan Guner is a Ph.D. student at the UNAM Materials Science and Nanotechnology Program at Bilkent University. His research interests are in the field of design and implementation of plasmon resonance sensing systems.

Erol Ozgur is a post-doctoral research associate at the UNAM Materials Science and Nanotechnology Program at Bilkent University. He currently works on large scale production and various applications of photonic and plasmonic biosensors.

Guzin Kokturk is a researcher at the UEKAE - BILGEM - The Scientific and Technological Research Council of Turkey (TUBITAK). She works in Bioelectronics Devices and Systems Group. Her research interests are development of biosensor devices and biological assays for different biosensor application areas.

Mehmet Celik is a Ph.D. student at the Department of Computer Engineering at Middle East Technical University. His research interests are in the field of computer vision and machine learning.

Elif Esen is researcher at the UEKAE - BILGEM - The Scientific and Technological Research Council of Turkey (TUBITAK). She is a Ph.D. student at Molecular Biology, Genetics and Biotechnolgy program at the Istanbul Technical University and continues to work in the fields of biosensor device and sensor chip development.

Ahmet Emin Topal is a Ph.D. student at the UNAM Materials Science and Nanotechnology Program at Bilkent University. His research interests are in the field of colorimetric biomolecular sensing and imaging using plasmon resonances.

Sencer Ayas is a post-doctoral fellow at the Canary Center at Stanford for Cancer Early Detection, Department of Radiology, Stanford School of Medicine. His research is on the design and realization of plasmonic nanostructures and metasurfaces, with applications to imaging and spectroscopy.

Yildiz Uludag is the Head of Computational Biology And Security Applications Unit at the UEKAE - BILGEM - The Scientific and Technological Research Council of Turkey (TUBITAK). She received her Ph.D. from Cranfield University (UK) and continues to work in the fields of biosensor device and sensor chip development.

Caglar Elbuken is Assistant Professor at Bilkent University, National Nanotechnology Research Center. His research interests include lab-on-a-chip devices, microdroplet-based microfluidic systems and sensing technologies for portable applications.

Aykutlu Dana is Associate Professor at Bilkent University, National Nanotechnology Research Center. He received his Ph.D. from Stanford University and continues to work in the fields of nanoscale optoelectronic devices and sensors. 